\title{
Optimal Performance Enhancement of Capacitor in Radial Distribution System Using Fuzzy and HSA
}

\author{
${ }^{1}$ P. Suresh Babu, ${ }^{2}$ B.Pamuleti, ${ }^{3}$ Dr M.Padma lalitha \\ ${ }^{1}$ Assistant Professor, Department of Electrical Engineering, Annamacharya Institute of Technology and \\ Sciences, Rajampet. \\ ${ }^{2}$ M.Tech. Student, Department of Electrical Engineering, Annamacharya Institute of Technology and Sciences, \\ Rajampet. 516216 (INDIA), \\ ${ }^{3}$ Professor, Head of the Department of Electrical Engineering, Annamacharya Institute of Technology and \\ Sciences, Rajampet.
}

\begin{abstract}
This paper presents a new two-stage methodology using the fuzzy and advanced Harmony search algorithm (HSA) for the placement of optimal capacitors on the primary feeders of the radial distribution systems with an objective to reduce the power losses and to improve the voltage profile. In the first stage, fuzzy approach is used to find the optimal capacitor locations and in the second stage, An advanced Harmony search algorithm is used to find the optimal sizes of the capacitors in the optimal capacitor locations identified by using the fuzzy approach. The proposed method is tested on 15-bus, 33-bus, 34-bus and 69-bus test systems and the results are presented.
\end{abstract}

Keywords: distribution systems, optimal capacitor placement, fuzzy approach, advanced harmony search algorithm.

\section{Introduction}

Radial distribution feeders are spread over large urban or rural areas and are responsible for a significant portion of total power loss. Optimal placement of shunt capacitors on the primary distribution feeders can results in reactive power compensation, improved voltage profile, power factor correction, power loss reduction as well as power quality improvement.

The objective of the capacitor placement problem is to determine the locations and sizes of the capacitors so that the power loss is minimized. Even though considerable amount of research work was done in the area of optimal capacitor placement [1 to 12], there is still a need to develop more suitable and effective methods for the optimal capacitor placement.

Some of the methods used for the optimal capacitor placement problem are efficient. Their efficiency entirely depends on the goodness of the data used. Fuzzy approach provides a remedy for any lack of uncertainty in the data. Fuzzy approach has the advantage of including heuristics and representing engineering judgments into the optimal capacitor placement problem. The solutions obtained from a fuzzy approach can be easily analyzed to determine optimal capacitor locations.

The global optimization method is more useful in obtaining the optimal capacitor sizes. Harmony search algorithm (HSA) is one of the new meta-heuristic methods in all the engineering fields [13 to 16].

In the first stage, fuzzy approach is used to find the optimal capacitor locations. In the second stage, Harmony Search Algorithm (HSA) is used to find the optimal sizes of the capacitors. The proposed method is tested on 15-bus, 33-bus, 34-bus, and 69-bus test systems and the results are presented.

\section{Problem Formulation}

The total real power loss $\left(\mathrm{P}_{\mathrm{L}}\right)$ in a distribution system having $n$ number of branches is given by:

$\sum_{i=1}^{n} I_{i}^{2} R_{i}$

Here $\mathrm{I}_{\mathrm{i}}$ is the magnitude of the branch current and $\mathrm{R}_{\mathrm{i}}$ is the resistance of the $i^{\text {th }}$ branch, respectively. The branch current can be obtained from the load flow solution. The branch current has two components, active component $\left(I_{a}\right)$ and reactive component $\left(I_{r}\right)$. The loss associated with the active and reactive components of branch currents can be written as: 


$$
\begin{aligned}
& \mathrm{P}_{\mathrm{La}}=\sum_{i=1}^{n} I_{a i}^{2} R_{i} \\
& \mathrm{P}_{\mathrm{Lr}}=\sum_{i=1}^{n} I_{r i}^{2} R_{i}
\end{aligned}
$$

For a single-source radial network, the loss $\mathrm{P}_{\mathrm{La}}$ associated with the active component of branch currents cannot be minimized because all active power must be supplied by the source at the root bus. However, supplying part of the reactive power demand locally can minimize the loss $\mathrm{P}_{\mathrm{Lr}}$ associated with the reactive component of branch currents. This paper presents a method that minimizes the loss due to the reactive component of the branch current by optimally placing the capacitors and thereby reduces the total loss in the distribution system.

\section{Identifiacation Of Optimal Locations Using Fuzzy Approach}

Two objectives are considered while designing a fuzzy approach for identifying the optimal capacitor locations. The two objectives are: (i) to minimize the real power loss and (ii) to maintain the voltage within the permissible limits. Voltages and power loss indices of distribution system nodes are modeled by fuzzy membership functions. A fuzzy inference system (FIS) containing a set of rules is then used to determine the capacitor placement suitability of each node in the distribution system. Capacitors can be placed on the nodes with the highest suitability.

In the first step, load flow solution for the original system is required to obtain the real and reactive power losses. Again, load flow solutions are required to obtain the power loss reduction by compensating the total reactive load at every node of the distribution system. The loss reductions are then, linearly normalized into a (0-1) range with the largest loss reduction having a value of 1 and the smallest one having a value of 0 . Power Loss Index value for $\mathrm{n}^{\text {th }}$ node can be obtained using equation 4.

$$
\begin{aligned}
& P L I(n) \frac{\text { Lossreduct ion }(n)-\text { Lossreduct ion }}{(\text { Lossreduct ion }(\max ))-\text { Lossreduct ion }} \\
& =
\end{aligned}
$$

These power loss reduction indices along with the p.u. nodal voltages are the inputs to the Fuzzy Inference System (FIS), which determines the node more suitable for capacitor installation.

In this paper, two input and one output variables are selected. Input variable-1 is power loss index (PLI) and Input variable-2 is the per unit nodal voltage (V). Output variable is capacitor suitability index (CSI) . Power Loss Index range varies from 0 to 1, P.U. nodal voltage range varies from 0.9 to 1.1 and Capacitor suitability index range varies from 0 to 1 . Five membership functions are selected for PLI. They are L, LM, M, $\mathrm{HM}$ and $\mathrm{H}$. All the five membership functions are triangular as shown in Figure-1. Five membership functions are selected for voltage. They are L, LN, N, HN and $\mathrm{H}$. These membership functions are trapezoidal and triangular as shown in Figure-2. Five membership functions are selected for CSI. They are L, LM, M, HM and $\mathrm{H}$. These five membership functions are also triangular as shown in Figure-3.

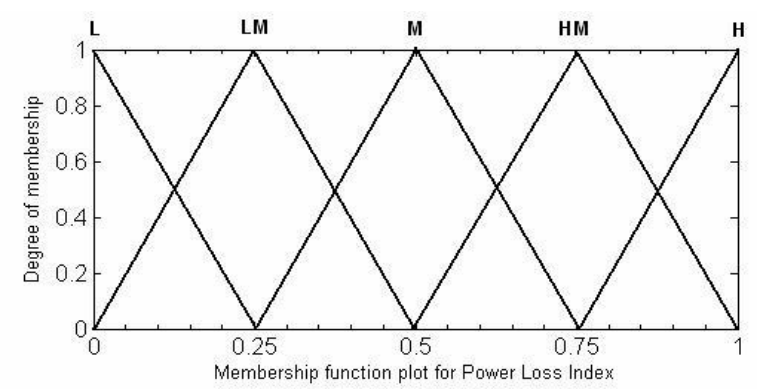

Figure-1. Membership function plot for P.L.I. 


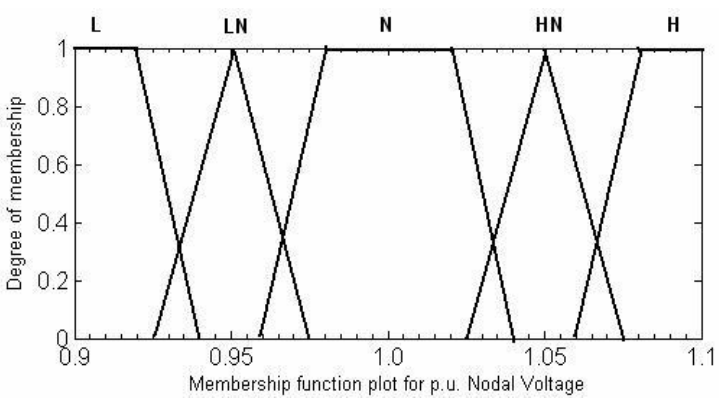

Figure-2. Membership function plot for p.u. nodal voltage.

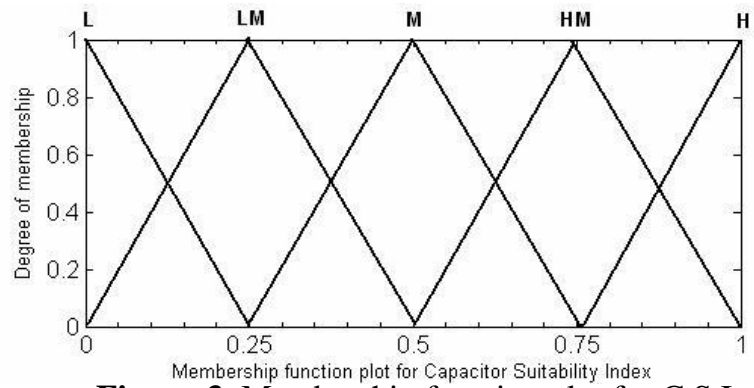

Figure-3. Membership function plot for C.S.I.

For determining the suitability of capacitor placement at a particular node, a set of multiple-antecedent fuzzy rules has been established. The inputs to the rules are the voltage and power loss indices and the output is the suitability of capacitor placement. The rules are summarized in the fuzzy decision matrix in Table -1 . The consequents of the rules are in the shaded part of the matrix. Optimal capacitor locations are identified based on the highest capacitor suitability index values.

Table-1. Decision matrix for determining the optimal capacitor locations.

\begin{tabular}{|c|c|c|c|c|c|c|}
\hline \multicolumn{2}{|c|}{ AND } & \multicolumn{5}{|c|}{ Voltage } \\
\hline & & $\mathbf{L}$ & $\mathbf{L N}$ & $\mathbf{N}$ & HN & H \\
\hline \multirow{5}{*}{$\overrightarrow{2}$} & L & LM & LM & $\mathrm{L}$ & $\mathrm{L}$ & L \\
\hline & LM & M & LM & LM & $\mathrm{L}$ & L \\
\hline & M & HM & $\mathrm{M}$ & LM & L & L \\
\hline & HM & HM & $\mathrm{HM}$ & $\mathrm{M}$ & LM & L \\
\hline & $\mathrm{H}$ & $\mathrm{H}$ & $\mathrm{HM}$ & M & LM & LM \\
\hline
\end{tabular}

\section{Harmony Search Algorithm}

The harmony search algorithm (HSA) is a new meta-heuristic algorithm [13 to 16]. The harmony search algorithm (HSA) is simple in concept, few in parameters and easy in implementation. Harmony search algorithm is concept from natural musical performance processes. The musicians starting with some harmonies, they attempt to achieve better harmonies by improvisation and create iteratively new good solutions based on past solutions on random modifications. Finally HSA gives optimum value.

This algorithm was originally developed for discrete optimization and later expanded for continuous optimization. It has been successfully applied to various computational optimization problems such as structural design, water network design, dam scheduling, school bus routing, Sudoku game, music composition, benchmark and real-world problems.

The HS algorithm initializes the Harmony Memory (HM) with randomly generated solutions. The number of solutions stored in the HM is defined by the Harmony Memory Size (hms). Then iteratively a new solution is created as follows. Each decision variable is generated either on memory consideration and a possible additional modification, or on random selection. The parameters that are used in the generation process of a 
new solution are called Harmony Memory Considering Rate (HMCR) and Pitch Adjusting Rate (PAR). After a new solution has been created, it is evaluated and compared to the worst solution in the HM. If its objective value is better than that of the worst solution, it replaces the worst solution in the HM. This process is repeated, until a termination criterion is fulfilled.

\section{Algorithm to find the capacitor sizes using harmony search algorithm}

After identifying the $n$ number of optimal capacitor locations using fuzzy approach, the capacitor sizes in all these $n$ optimal locations are obtained by using the Harmony Search Algorithm.

Step 1: Initialize all the parameters and constants of the Harmony search algorithm. They are $\mathrm{Q}_{\mathrm{Cmin}}, \mathrm{Q}_{\mathrm{Cmax}}$, (Minimum and maximum limits for Capacitor sizes), hms,

$\mathrm{HMCR}, \mathrm{PAR}_{\min }$ and $\mathrm{PAR}_{\max }$.

Step 2: Run the load flow program and find the total real

power loss $\mathrm{P}_{\mathrm{Loss} 1}$ of the original system. (Before capacitor placement)

Step 3: Initialize the harmony memory i.e., generate $[h m s \mathrm{x} n]$ number of initial solutions randomly within the limits, where $h m s$ is the harmony memory size and $n$ is the number of capacitors. Each row represents one possible solution to the optimal capacitor-sizing problem. For example; $n=1$ for one capacitor, $n=2$ for two capacitors, $n=3$ for three capacitors, $n=4$ for four capacitors and $n=5$ for five capacitors and $h m s=10$ for ten harmony, $h m s=20$ for twenty harmony, $h m s=50$ for fifty harmony, $h m s=100$ for hundred harmony and $h m s$ $=200$ for two hundred harmony.

Step 4: Place all the $n$ capacitors of the harmony vector i.e., each row of the Harmony vector at the respective optimal capacitor location and perform the load flow

analysis and find the total real power loss $\mathrm{P}_{\mathrm{Loss} 2}$ and then obtain the loss reduction (fitness value) using equation (5)

Fitness Value $=\mathrm{P}_{\text {Loss1 }}-\mathrm{P}_{\text {Loss2 }}$

Repeat the same procedure for all the rows of the harmony vector to find Fitness values.

Step 5: Obtain the best fitness value by comparing all the fitness values.

Step 6: Start the improvisation (Iteration count is set to one).

Step 7: Improvisation of the New Harmony is generating a new harmony.

A New Harmony vector is generated based on the following steps:

(i) Random selection: It is used to select one value randomly for a certain element of the new vector from the possible range $\left(\mathrm{Q}_{\mathrm{Cmin},} \mathrm{Q}_{\mathrm{Cmax}}\right)$ of values.

(ii) Memory consideration: It is used to choose the value for a certain element of the new vector from the specified HM range.

$\mathrm{X}_{\mathrm{i}_{\mathrm{i}}}=\mathrm{x}_{\mathrm{i}}^{\prime} \in\left\{\mathrm{x}_{1}^{\prime}, \mathrm{x}_{2}^{\prime}, \ldots \mathrm{x}_{\mathrm{HMS}}^{\mathrm{HM}}\right\}$ with probability HMCR (6)

$\mathrm{x}_{\mathrm{i}}^{\prime}{ }_{\mathrm{x}^{\prime} \mathrm{i}} \in \mathrm{X}_{\mathrm{i} \text { with }}$ probability (1-HMCR)

Step 8: Pitch adjustment: It is used to adjust the values of the New Harmony vector obtained in step 7. (Between $\mathrm{PAR}_{\min }$ and $\mathrm{PAR}_{\max }$ ).

$\mathrm{x}_{\mathrm{i}}^{\prime}=\mathrm{x}_{\mathrm{i}}^{\prime} \pm \operatorname{rand}(0,1) * \mathrm{bw}$

(bw - band width varies between a higher value and a lower value from first iteration to last iteration)

Step 9: Find the fitness values corresponding to the New Harmony generated and pitch adjusted in steps 7 and

8. Step 10: Apply Greedy Search between old harmony and New Harmony by comparing fitness values.

Step 11: Update harmony memory, by replacing the worst harmony with the new best Harmony. Obtain the best fitness value by comparing all the fitness values.

Step 12: The improvisation (iteration) count is incremented and if iteration count is not reached maximum then go to step 7 .

Step 13: The solution vector corresponding to the best fitness value gives the optimal capacitor sizes in $n$ optimal locations. 


\section{Results}

Fuzzy approach is used to find the optimal capacitor locations and HSA is used to find the optimal capacitor sizes. In this present work, $\mathrm{hms}=30, \mathrm{HMCR}=85 \%$, No of improvisations $=200, \mathrm{PAR}_{\min }=0.4$ and $\mathrm{PAR}_{\max }=0.9$.

\subsection{Results of 15-bus system}

The proposed algorithm is applied to 15-bus system [17]. Optimal capacitor locations are identified based on the C.S.I. values. For this 15-bus system, five optimal locations are identified. Capacitor sizes in the five optimal locations, total real power losses before and after compensation are shown in Table-2.

Table-2. Results of 15-bus system.

\begin{tabular}{|c|c|}
\hline Bus No. & Capacitor size in kVAr \\
\hline 4 & 345 \\
\hline 6 & 265 \\
\hline 7 & 143 \\
\hline 11 & 300 \\
\hline 15 & 143 \\
\hline Total kVAr & 1196 \\
\hline Total real power loss in \\
kW (before) & 61.7944 \\
\hline Total real power loss in \\
$\mathrm{kW}$ (after)
\end{tabular}

\subsection{Results of 33-bus system}

The proposed algorithm is applied to 33 bus system [18]. Optimal capacitor locations are identified based on the C.S.I. values. For this 33-bus system, one optimal location is identified. Capacitor sizes in one optimal location, total real power losses before and after compensation are shown in Table-3.

Table-3. Results of 33-bus system.

\begin{tabular}{|c|c|}
\hline Bus No. & $\begin{array}{c}\text { Capacitor size in } \\
\text { kVAr }\end{array}$ \\
\hline 30 & 1315 \\
\hline Total kVAr & 1315 \\
\hline $\begin{array}{c}\text { Total real power loss in } \mathrm{kW} \\
\text { (before) }\end{array}$ & 369.2558 \\
\hline $\begin{array}{c}\text { Total real power loss in } \mathrm{kW} \\
\text { (after) }\end{array}$ & 297.9886 \\
\hline Loss reduction & 71.2672 \\
\hline \% Loss reduction & $19.30 \%$ \\
\hline
\end{tabular}

\subsection{Results of 34-bus system}

The proposed algorithm is applied to 34-bus system [7]. Optimal capacitor locations are identified based on the C.S.I. values. For this 34-bus system, seven optimal locations are identified. Capacitor sizes in the seven optimal locations, total real power losses before and after compensation are shown in Table-4. 
Table-4. Results of 34-bus system.

\begin{tabular}{|c|c|}
\hline Bus No. & Capacitor size in kVAr \\
\hline 20 & 968 \\
\hline 21 & 145 \\
\hline 22 & 144 \\
\hline 23 & 143 \\
\hline 24 & 143 \\
\hline 25 & 143 \\
\hline 26 & 228 \\
\hline Total kVAr & 1914 \\
\hline Total real power loss in \\
kW (before)
\end{tabular}

\subsection{Results of 69-bus system}

The proposed algorithm is applied to 69-bus system [4]. Optimal capacitor locations are identified based on the C.S.I. values. For this 69-bus system, two optimal locations are identified. Capacitor sizes in the two optimal locations, total real power losses before and after compensation are shown in Table-5.

Table-5. Results of 69-bus system.

\begin{tabular}{|c|c|}
\hline \multicolumn{1}{|l|}{ Bus } & Capacitor size in kVAr \\
\hline 61 & 1123 \\
\hline 64 & 207 \\
\hline Total kVAr & 1330 \\
\hline Total real power loss in \\
kW (before) & 225.0044 \\
\hline Total real power loss in & \\
\hline kW (after) & 151.7074 \\
\hline Loss reduction & 73.2970 \\
\hline \% Loss reduction & $32.58 \%$ \\
\hline
\end{tabular}

\section{Conclusions}

In this paper, a two-stage methodology of finding the optimal locations and sizes of shunt capacitors for loss reduction in radial distribution systems is presented. Fuzzy approach is proposed to find the optimal capacitor locations and Harmony Search Algorithm is proposed to find the optimal capacitor sizes. Based on the simulation results, the following conclusions are drawn:

By installing shunt capacitors at all the optimal locations, the total real power loss of the system has been reduced significantly and bus voltages are improved substantially. The fuzzy approach is capable of determining the optimal capacitor locations based on the C.S.I. values. The proposed Harmony Search Algorithm iteratively searches the optimal capacitor sizes corresponding to minimum loss. 


\section{References}

[1] Duran H. 1968. Optimum number, location and size of shunt capacitors in radial distribution feeders: A dynamic programming approach. IEEE Transactions on Power Apparatus and Systems. 87(9): 1769-1774, September.

[2] Bae Y.G. 1978. Analytical method of capacitor allocation on distribution primary feeders. IEEE Transactions on Power Apparatus and Systems. PAS-97(4): 1232-1238, July.

[3] Grainger J.J and S.H. Lee. 1981. Optimum size and location of shunt capacitors for reduction of losses on distribution feeders. IEEE Transactions on Power Apparatus and Systems. PAS-100(3): 1105-1118, March.

[4] Baran M.E. and Wu F.F. 1989. Optimal capacitor placement on radial distribution systems. IEEE Transactions on Power Delivery. 4(1): 725-734, January.

[5] Baran M.E. and Wu F.F. 1989. Optimal sizing of capacitors placed on a radial distribution system. IEEE Transactions on Power Delivery. 4(1): 735-743, January.

[6] Sundhararajan S. and Pahwa A. 1994. Optimal selection of capacitors for radial distribution systems using a genetic algorithm. IEEE Transactions on Power Systems. 9(3): 1499-1507, August.

[7] Chis M., Salama M.M.A. and Jayaram S. 1997. Capacitor placement in distribution systems using heuristic search strategies. IEE proceedings on Generation, Transmission and Distribution. 144(3): 225-230, May.

[8] Haque M.H. 1999. Capacitor placement in radial distribution systems for loss reduction. IEE Proceedings on Generation, Transmission and Distribution. 146(5): 501-505, September.

[9] Ng H.N., Salama M.M.A. and Chikhani A.Y. 2000. Capacitor allocation by approximate reasoning: fuzzy capacitor placement. IEEE Transactions on Power Delivery. 15(1): 393-398, January.

[10] Prakash K. and Sydulu M. 2007. Particle swarm optimization based capacitor placement on radial distribution systems. IEEE Power Engineering Society general meeting. pp. 1-5, June.

[11] M. Damodar Reddy and V.C. Veera Reddy. 2008. Optimal capacitor placement using fuzzy and real coded genetic algorithm for maximum savings. Journal of Theoretical and Applied Information Technology. 4(3): 219-226.

[12] M. Damodar Reddy and V.C. Veera Reddy. 2008. Capacitor placement using fuzzy and particle swarm optimization method for maximum annual savings. ARPN Journal of Engineering and Applied Sciences. 3(3): 25-30.

[13] Z. W. Geem, J. H. Kim and G. V. Loganathan. 2001. A new heuristic optimization algorithm: harmony search. Simulation. 76(2): 6068 .

[14] K.S. Lee and Z.W. Geem. 2004. A new meta-heuristic algorithm for continues engineering optimization: harmony search theory and practice. Computer methods in applied mechanics and Engineering. 194: 3902-3933.

[15] R. Srinivasa Rao. 2010. A Hybrid Approach for Loss Reduction in Distribution Systems using Harmony Search Algorithm. International Journal of Electrical and Electronics Engineering. 4: 7.

[16] R. Sirjani, A. Mohamed and H. Shareef. 2010. Optimal capacitor placement in a radial distribution system using harmony search algorithm. Journal of Applied Sciences. 10(23): 2998-3006.

[17] Das D., Kothari D.P. and Kalam A. 1995. Simple and efficient method for load flow solution of radial distribution networks. Electrical Power and Energy Systems. 17(5): 335-346.

[18] Baran M.E. and Wu F.F. 1989. Network reconfiguration in distribution systems for loss reduction and load balancing. IEEE Transactions on Power Delivery. 4(2): 1401-1407, April. 